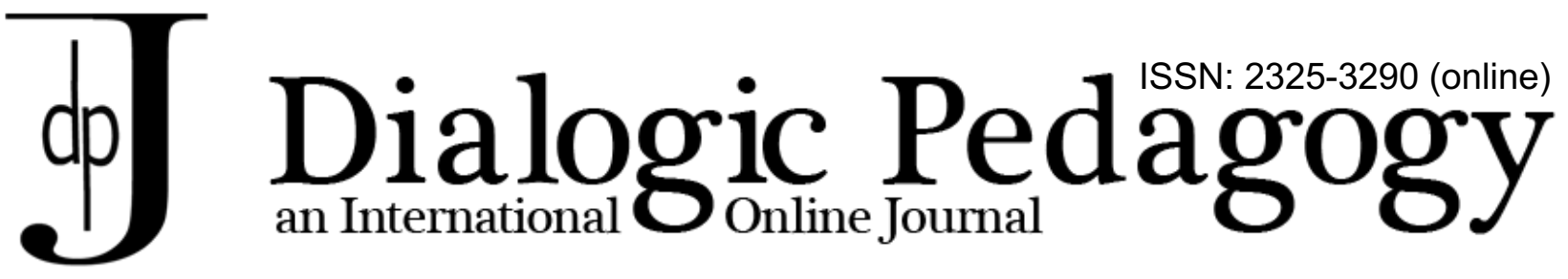

\title{
Whose Discourse, Whose Ears? Harmony in Dialogic Pedagogy amidst the Post-Truth Noise
}

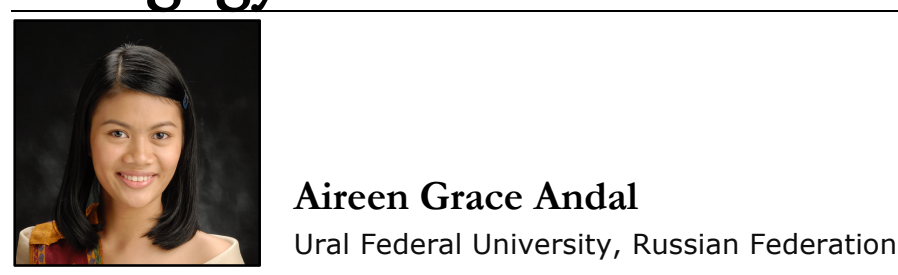

\begin{abstract}
Commentary on DPJ Editorial by Robin Alexander (2019), Whose discourse? Dialogic Pedagogy for a post-truth world. This commentary adds emphasis on the importance of the four areas of dialogic pedagogy--language, voice, argument and truth-- that Alexander proposes to be invested in and prioritized more. It is argued that dialogic pedagogy will benefit from the development of the current approach to respond to the post-truth era, rather than from looking for new ways to do dialogue. Finally, it is suggested that practitioners of dialogic pedagogy take the post-truth era as a situation that fosters critical thinking and reevaluation of how dialogue is conducted.
\end{abstract}

Aireen Grace Andal is a graduate student of political philosophy and a junior researcher at the Center for Comparative Studies on Toleration and Recognition at the Ural Federal University. She is a citizen of the Philippines, where she obtained her bachelor's degree in sociology. Her recent fields of research interests are ethics, international relations and urban spaces.

\section{Acknowledgement}

The author thanks Prof. Andrey Menshikov whose mentorship in class inspired this commentary and the teachers in Global Ambassador, under the leadrship of Evgeniya Efremova, for their thoughtful discussions about pedagogy.

$\cos 0300$

\section{Introduction}

The article by Dr. Alexander (2019) serves as an invitation to educators and students to engage in a dialogue about the assumptions held regarding virtues of dialogue within the classroom and the broader world. This is especially pertinent to the contemporary era where dialogue enters the post-truth world. There is indeed danger for this changing status of truth to drown out, restrict, or confuse our understanding of what a classroom dialogue is, and why and how to do it. The opportunity cost of the tendency to be unreflective about dialogue is the dearth of understanding and exchanges of thoughts particularly because of a limited take on the normative pursuit of dialogue. How should we make sense of these realities? Given the promises of dialogic pedagogy, why has this not been enough to combat the post-truth threats? Dr. Alexander offered ways of looking at the current state of dialogic pedagogy. He showed the possibilities of having discord and incoherence in the virtues and practices of dialogic pedagogy in the classroom. More importantly, he demonstrated not only how dialogue should be done but how it is done poorly in the post- 
truth era. This commentary takes stock of what Dr. Alexander has started and interprets his suggestions as a call not to do something new but to scale up what is already being done in dialogic pedagogy.

\section{On language: We don't pause enough}

Dr. Alexander's proposal to ensure an "accountability to talk" means to see the spill-over of these talks outside the classroom. Dialogues inside the classroom are pointless if there is a "mismatch between what we strive for within classrooms and what students may encounter outside them" (p. E6). This renders futile the speech norms developed in the classroom dialogues because they do not trickle down into the students' ability to reflect how their dialogue is connected to the larger society they live in. Of no less importance are the unuttered interactions such as signs of doubts, nods of insights, pauses of reconsideration etc. These provide assistance to an accountable talk, making it impossible to think of dialogues as beyond an expression of logic, but as a reflection about the participants as well as the positions they take. Without leaving rigid polarisations, dialogue very quickly lapses into a rather dogmatic practice.

The challenge for educators then is to offer more opportunities for students to pause and engage into analyzing why they speak, not only what they have to speak about and how to do it. It is important because what use is it if students are able to speak yet the dialogues are mostly still characterised by the discourses of dichotomies, myths and meaninglessness? If dialogue is "the object of learning as well as its medium" (p. E6), students would be able to generate a shared lexicon to coexist in a world wherein metadebates are the norm for legitimate dialogue. This reminds us of the role of language as means not only to offer facts, but also to unveil the backdrop that prevents students from seeing otherwise.

\section{On Voice: We don't hush enough}

Voice, by itself, may not be sufficient to cure dialogue's deficit. Dialogic pedagogy is an approach that weighs positions, not just surfaces them. Even where engaging and highly critical classroom dialogues emerge, these have little bearing if the noise of technocratic "expert" voices drown out the very voices of the people they wish to educate. This reflects a hierarchy of voices and an ableist classroom to which Alexander commented that "while freedom is never absolute it can be tempered" (p. E8). The previous statement resembles what Shanahan (2007) meant in saying that "children may have voices, but adults control the conversation" (p. 415). To be dialogic is not simply to allow students to talk in more volume but to actually listen to these voices. For the muted voices to be heard, the noise of the dominant voices must be controlled. Not only teachers must scale down their controlling of the conversations, but also teachers should scale up controlling their own voices as well as other dominant voices.

Silence, while seemingly ironic, is an enabling tool to create "intermissions" for other voices to begin rising up. If this becomes a routine, while dominant voices become minor voices and observers, these dominant voices can listen to themselves from way above and then hopefully come to realize how caught up they have been as dominant ones, driving other voices out of stage. As Alexander reminds us, "we must ask how far it equalises the voices of different groups of students" (p. E10). Sometimes, it is even helpful to mute one's own voice and engage in dialogues assuming others' voices. For instance, Plato had dialogues through a character. He was able to engage with the ideas and enter into a pedagogical relationship with his audience. A dialogue is therefore not weighed in terms of flaunting a range of assertions at one's disposal, though that might be useful; but rather, in terms of demonstrating kind of reasoning that is sensitive not only to valid premises and conclusions but also to listening to various sources of voices in an argument. 


\section{On argument: We don't freestyle enough}

The merit of an argument is not in its flawlessness but in its power to integrate and establish connection among various fields of life. However, we are obsessed with rigid and clean-cut categories such as types, kinds or groups. But as Alexander mentions, we do a great disservice to arguments if we are not able to transcend the boundaries of the academic and political realms. Arguments are products of thought processes that organize the premises to makes sense of any interpretation of reality. While dialogue enables many of us to carefully reflect on our own predispositions, it can backfire if we continue "polarising academic and political argument as if each were one-dimensional" (p. E11). If educators are to fulfil their purpose, they cannot afford to ignore that arguments in the academe are not only integrated with the political world but that these two are inevitably permeable membranes. Arguments build communities of dialogue, spaces where students can explore further what they were taught and think for themselves about it. Taken in these terms, arguments are more than discrete skills learned in schools but an approach to learning that sees the blurred lines between academe and politics.

As implied in Dr. Alexander's text, it would be a sad fact if arguments are determined by labels academic, political, moral etc. If arguments are polarized, the dialogues in the classroom would be framed, down to the last detail, by the requirement to prepare for academic requirements rather than the broader world. When students lay down their arguments, the focus will not be on category-free discussions or enquiry, but on choosing arguments based on what we need to know for the school requirements. This situation is not far from the traditional classroom experience and military training ground, where students are trained to produce arguments that are favorable towards fulfilling requirements. To add to Alexander's point, part of this concern is when educators and learners remain passive and "mechanical dialogue" is left unchallenged. Worst is when there is denial that being consumed by categories is an impediment to learning. As what Wittgenstein said, "[t]elling someone something he will not understand is pointless" (Wittgenstein, 1998, p. 10). What are some signs of this "mechanical dialogue"? On the part of educators, grades are introduced as goals, and obedience as the means. Ranking is emphasized, which is why students focus on winning debates rather than appreciating their opponents' views. The teacher might also essentialize his or her role as an authority, instigating automatic obedience from students. There is less emphasis on the long-term influences that the school experience can have on students' beliefs. On the part of students, they may fail to see the link between their present learning and its critical consequences for society in the future. There is also no more sense of wonder, and even confusion, and the desire to get beyond mere papers and records. In this situation, there rarely emerges learning from the collision of viewpoints.

\section{On Truth: We don't sustain enough}

Dialogic pedagogy can be easily dismissed in its futile pursuit of truth if by 'truth' we mean a kind of compatibility of knowledge claims with a universal reality. Unsurprisingly, those who support the "cynical relativism of 'fake news' and the 'alternative fact'" (Alexander, 2019, p. E13) may not find dialogue to be the most supportive space. In other words, post-truth advocates have inherent cognitive limitations in processing a dialogic rhetoric. This is because they, by design, are not ready to celebrate dialogue. Yet this phenomenon of truth denial cannot be easily relinquished especially in discursive venues such as those found online, or in assemblies of post-truth supporters who provide hospitable platforms for truth-assaultive discourses. No less bothersome than believing in post-truth is when the lack of trust to the pursuit of truth compounds to apathy. Even in the presence of the well-facilitated dialogues, we have to recognise that people may plainly not care about the process of obtaining information. Apathy in regard to pursuing the truth cannot be isolated from the fact that people typically rely on knowledge presented by the key informants and that many have simply had enough of "truth." 


\section{Whose Discourse, Whose Ears? Harmony in Dialogic Pedagogy amidst the Post-Truth Noise}

Aireen Grace Andal

The challenge therefore is not so much about producing counterclaims to post-truth than it is about proactively sustaining the spaces that celebrate truth. If anything, what dialogic pedagogy is after in its pursuit of truth is to foster and maintain negotiations of knowledge claims and being able to express truth in various ways. It may be easy to spark activities that foster dialogic pedagogy. But the challenge is to keep the dialogues going, to keep the contesting positions, to keep asking questions. To dismiss sustaining what has begun in dialogic pedagogy is to judge it by a limited standard for which it was established in the first place. It would be inapt to ignore the difference between truth as claim and truth as a heuristic device, a way to facilitate dialogue and negotiation. The aim is to avoid monopoly of knowledge production. This does not imply that every viewpoint is equally valuable, or that we should accept that each person finds their own truth. Rather, the logic of negotiation primes participants to be broad-minded and other-regarding.

\section{Harmony amidst the Post-Truth Noise}

It is in this context of much discord that dialogic pedagogy finds itself today. While there are definitely efforts to keep dialogic pedagogy active especially in the digital age, there are reasons to doubt what these ventures can achieve. Has dialogic pedagogy ended up with a promising ideal overwhelmed by the whims of politics of knowledge production? Yet while the post-truth craze mocks and invalidates the normative aspirations of dialogic pedagogy, it is this situation that urges us to explore further what could be done better in dialogic pedagogy. It is the disruptive noise of the post truth era that makes us hungry to revisit why and how we practice dialogue, that challenges our current dialogic practices, that nudges us away from dogmatic beliefs that impedes critical thought. The post-truth apologists are of great value to dialogue even when their beliefs are barren at best, and harmful at worst. This prevents the situation that worried Mill (1859), that is, "both teachers and learners go to sleep at their post, as soon as there is no enemy in the field" (p. 44).

The takeaway in Dr. Alexander's account is the challenge to scale up dialogue and produce melody amidst the post-truth noise. Perhaps there is no need to do dialogue differently, only to play it better. The question is not so much about looking for new answers than it is about listening to the often ignored and skipped aspects of dialogue in the classroom. The challenge is to cultivate the art of listening or a sensitivity to the signals when to slow down, stop, silence and adjust classroom dialogues as integrated to the rest of the world. This is akin to recognizing the notes played before a concert begins, joining all instruments of the orchestra to play in consummate connectedness around a common frequency. Certainly, it is inevitable to experience an inadequacy to perform this. However, Dr. Alexander offered four ways to practice mindfulness or consciousness on a seamless integration of doing and being in the practice of dialogic pedagogy. Like an embedded antenna that allows an examination of our inner and outer environment, sensitivity to the connection of classroom dialogues to its outside environment makes a difference.

Dialogic pedagogy could be easily dismissed as an illusion with no bearing on the world of practical education especially when dealing with lack of classrooms or underpaid teachers. The post-truth supporters can easily lurk over the impracticality and non-immediacy of dialogic pedagogy. What then is the point of all the attempts to revisit the ways we conduct dialogue? The value of it lies in creating enough confidence in the existence of a logical way of arriving at any epistemic gain. The pursuit of a well-designed dialogue means a careful weighing of various levels of talk among educators and students. Here, the goal is not necessarily consensus but a legitimate way to process knowledge claims so that "information of the highest possible quality can be obtained" (Funtowicz \& Strand, 2007). In order for a legitimate dialogic process to take place, parties must be able to identify the bases on which their agreements rest and consider it to be a product of a fair discussion where everyone had an equal chance of affecting the outcomes. It is through the distinct process of exchanging and carefully considering reasons that mutually-acceptable and legitimate classroom dialogues could be generated. This is an opening to amplify the application of 


\section{Whose Discourse, Whose Ears? Harmony in Dialogic Pedagogy amidst the Post-Truth Noise}

Aireen Grace Andal

dialogues as a response to the current context. The endeavors of dialogic pedagogy may have been the laughing stock for those who remain unconvinced by the legitimacy of exchanges as facilitated by reason. But it has also been a source of hope for those who keep on seeking to make pedagogy better by the pursuit of truth. Dialogic pedagogy has a lot more to offer, especially when we lend ears for possibilities to improve beyond the mere talk.

\section{References}

Alexander, R. (2019). Whose discourse? Dialogic Pedagogy for a post-truth world. Dialogic Pedagogy: An International Online Journal, 7, E1-E19. doi:https://doi.org/10.5195/dpj.2019.268

Funtowicz, S. O., \& Strand, R. (2007). Models of Science and Policy. In T. Traavik \& L. L. Ching (Eds.), Biosafety First (pp. 263-278). Trondheim: Tapir Academic Press.

Mill, J. S. (1859). On Liberty and Other Writings. In S. Collini (Ed.), Cambridge texts in the History of Political Thoughts. New York: Cambridge University Press.

Shanahan, S. (2007). Lost and found. The sociological ambivalence toward childhood. The Annual Review of Sociology, 33, 407-428. doi:10.1146/annurev.soc.33.040406.131808

Wittgenstein, L. (1998). Culture and Value: a Selection from the Posthumous Remains (P. Winch, Trans.). Oxford: Blackwell Publishers.

\section{(c)) EY}

New articles in this journal are licensed under a Creative Commons Attribution 4.0 United States License.

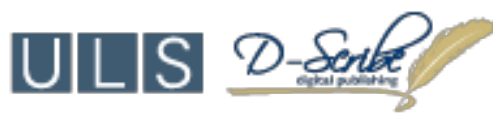

This journal is published by the University Library System, University of Pittsburgh as part of its D-Scribe Digital Publishing Program and is cosponsored by the University of Pittsburgh Press. 